\title{
Unilateral Storage of Fear Memories by the Amygdala
}

\author{
Hugh T. Blair, Virginia K. Huynh, Vanessa T. Vaz, John Van, Reekesh R. Patel, Amit K. Hiteshi, Jennie E. Lee, and \\ Jason W. Tarpley \\ Department of Psychology, University of California, Los Angeles, Los Angeles, California 90024
}

Pavlovian fear conditioning is an associative learning task in which subjects are trained to respond defensively to a neutral conditioned stimulus (CS) by pairing it with an aversive unconditioned stimulus (US). This type of learning depends critically on the amygdala, and evidence suggests that synaptic plasticity within the lateral nucleus of the amygdala (LA) may be responsible for storing memories of the CS-US association. In the present study, we trained rats to fear an auditory CS by pairing it with a shock US delivered to one eyelid. Conditioning was assessed by measuring freezing responses evoked by the CS during a subsequent test session. The amygdala was unilaterally inactivated during either the training or the testing session by intracranial infusions of muscimol into the LA. We found that both acquisition and expression of conditioned freezing to the CS depended on the amygdala contralateral but not ipsilateral from the eyelid where the shock US was delivered. To explain this surprising result, we propose that the shock US is relayed from the eyelid to the amygdala via lateralized nociceptive sensory pathways, which causes memories of the CS-US association to be stored by the amygdala contralateral but not ipsilateral from the shocked eyelid. Our results demonstrate that the fear-learning circuitry of the amygdala is functionally lateralized according to the anatomical source of predicted threats. In future studies, the cellular mechanisms of emotional memory storage might be pinpointed by identifying cellular processes that occur only in the amygdala contralateral but not ipsilateral from the US during lateralized fear conditioning.

Key words: hemispheric lateralization; conditioning; pain; muscimol; learning; memory

\section{Introduction}

Pavlovian fear conditioning is an associative learning task in which subjects are trained to fear a neutral conditioned stimulus (CS) paired with an aversive unconditioned stimulus (US). For example, rats can be trained to fear a neutral auditory tone (the CS) by pairing it with electric shock (the US). Before such pairing, the tone elicits very little response from the rat, but after pairing, the tone can elicit a variety of conditioned emotional responses (CERs) such as freezing, a potentiated startle reflex, distress calls, and autonomic changes in heart rate and blood pressure (LeDoux et al., 1988; Davis, 1992). The emergence of these CERs indicates that the rat has learned to fear the CS because it predicts the US.

To explain the emergence of these CERs during fear conditioning, it has been hypothesized that the CS becomes associated with an internal emotional state of fear that is elicited by the US (Konorski, 1967; Wagner and Brandon, 1989). The amygdala is thought to be critical for encoding such emotional fear states (Klüver and Bucy, 1937; Weiskrantz, 1956), and pavlovian fear conditioning is severely impaired when the amygdala is bilaterally damaged or destroyed (LeDoux et al., 1990a,b; Davis, 1992; Maren, 2001). Based on these and other findings, it has been

Received Aug. 24, 2004; accepted March 15, 2005.

This work was supported by a University of California, Los Angeles, new faculty startup grant (H.T.B.). We thank Joseph LeDoux, Peter Dayan, Tom Blair, Michael Fanselow, Bernard Balleine, and Josh Johansen for helpful discussions and comments on this manuscript and Adam Welday for technical assistance.

Correspondence should be addressed to Dr. Hugh T. Blair, Department of Psychology, University of California, Los Angeles, 1285 Franz Hall, Los Angeles, CA 90095-1563. E-mail: blair@psych.ucla.edu.

D0I:10.1523/JNEUROSCI.0674-05.2005

Copyright $\odot 2005$ Society for Neuroscience $\quad$ 0270-6474/05/254198-08\$15.00/0 hypothesized that the amygdala is a site of emotional memory storage where synaptic plasticity stores an association between the CS and US during fear conditioning (for review, see Blair et al., 2001).

In contrast with the severe impairment observed after bilateral amygdala lesions, unilateral amygdala lesions cause only mild impairment of conditioned freezing to an auditory CS in rats, with no significant difference between the left and right hemispheres (LaBar and LeDoux, 1996; Goosens and Maren, 2001; Baker and Kim, 2004). This suggests that each amygdala hemisphere can independently support auditory fear conditioning. However, contextual fear conditioning has been shown to depend more on the right than the left amygdala in rats (Baker and Kim, 2004). Human studies have also yielded evidence that the left and right amygdala can be asymmetrically activated by aversive stimuli depending on the stimulus modality (Funayama et al., 2001; Phelps et al., 2001) or whether the stimulus is consciously versus subconsciously perceived (Morris et al., 1998). In addition, evidence from human neuroimaging studies suggests that the emotional memory functions of the left versus right amygdala may differ in women versus men (Cahill et al., 2001, 2004; Canli et al., 2002).

Despite this abundance of evidence for lateralization of the emotional memory functions of the amygdala, no previous studies have investigated whether lateralization of fear representations in the amygdala depend on lateralization of the feararousing stimulus itself. In the present study, we investigated this question by training rats to fear an auditory CS paired with a unilateral shock US delivered to either the left or right eyelid (counterbalanced among rats). We found that acquisition and 
expression of CS-elicited freezing behavior depended only on the amygdala contralateral from the shocked eyelid and not on the ipsilateral amygdala. These results show for the first time that the fear-learning circuitry of the amygdala is functionally lateralized such that each amygdala hemisphere can independently process threats that are anticipated on the contralateral side of the body. We discuss the implications of this discovery for current theories and future investigations of the role of the amygdala in learning, memory, and emotions.

\section{Materials and Methods}

Subjects and surgery. Male Long-Evans rats weighing 350-400 g were reduced to $85 \%$ of their ad libitum weight through limited daily feeding. Under deep isoflurane anesthesia, they were bilaterally implanted with intracranial infusion cannulas (22 gauge guides) targeted at the lateral nucleus of the amygdala (LA) $(3.0 \mathrm{~mm}$ posterior, $\pm 5.3 \mathrm{~mm}$ lateral, and $8.0 \mathrm{~mm}$ dorsal to bregma) or at the overlying caudate for the offsite control group ( $3.0 \mathrm{~mm}$ posterior, $\pm 5.0 \mathrm{~mm}$ lateral, and $6.0 \mathrm{~mm}$ dorsal to bregma). Two silver wires ( $75 \mu \mathrm{m}$ diameter, stripped of insulation $2 \mathrm{~mm}$ from the tip) were threaded through the skin of each eyelid for delivery of the periorbital shock US. All animals received bilateral cannula implants (except for off-site controls in which cannulation was unilateral) and bilateral shock electrode implants, although some animals did not receive bilateral infusions or bilateral shocks during the experiment.

Fear conditioning. After at least $5 \mathrm{~d}$ of recovery from surgery, rats were preexposed for $5 \mathrm{~d}(15 \mathrm{~min} / \mathrm{d})$ to the experimental platform, and then the fear-conditioning experiment was conducted. Throughout preexposure and fear-conditioning sessions, rats constantly foraged for $20 \mathrm{mg}$ of purified food pellets dropped from an overhead dispenser at $\sim 30$ s intervals to provide a baseline of motor activity against which freezing behavior could easily be measured. The CS was a series of 20 white noise pips ( 80 $\mathrm{dB}$ ), each $250 \mathrm{~ms}$ in duration, presented at a rate of $1 \mathrm{~Hz}$ (total CS duration, $19.25 \mathrm{~s})$. The US was a train of 20 shock pulses $(2.5 \mathrm{~mA})$, each $2 \mathrm{~ms}$ in duration, delivered to the eyelid at a rate of $10 \mathrm{~Hz}$ (total US duration, $2 \mathrm{~s}$ ). For paired training (all groups except the unpaired drugfree control group), the US began $300 \mathrm{~ms}$ after the offset of the final CS pip. For unpaired training (the unpaired drug-free control group only), the US was delivered exactly halfway through the intertrial interval separating one trial from the next. The duration of the intertrial interval for both training and test sessions was uniformly randomized between 2 and 4 min on every trial. The rat's moment-to-moment position in the chamber was sampled at $60 \mathrm{~Hz}$ by an overhead video tracking system, which monitored the location of three light-emitting diodes (red, blue, and green) attached to the animal's head stage for automated scoring of freezing behavior, as described in previous studies (Moita et al., 2003, 2004).

Amygdala inactivation. Drugs were infused through 28 gauge injector cannulas attached to a $1.0 \mu \mathrm{l}$ Hamilton (Reno, NV) syringe via polyurethane tubing as described in a previous study (Wilensky et al., 1999). Into each hemisphere of the amygdala, we infused $0.5 \mu \mathrm{l}$ of muscimol dissolved at a concentration of $0.25 \mathrm{mg} / \mathrm{ml}$ in sterile $0.9 \%$ saline vehicle. The infusion rate was $0.25 \mu \mathrm{l} / \mathrm{min}$. After drug infusion, the cannulas were left in place for an additional $1 \mathrm{~min}$ to allow diffusion of the drug away from the cannula tip, after which the dummy cannulas were replaced.

\section{Results}

Fear-conditioning experiments were conducted while rats foraged for small food pellets on a $1 \mathrm{~m}^{2}$ square platform. After $5 \mathrm{~d}$ of preexposure to the platform, rats were conditioned by pairing a $20 \mathrm{~s}$ auditory CS with a $2 \mathrm{~s}$ shock train US delivered through electrode wires implanted in the skin beneath the eyelid. Conditioned fear was measured by detecting freezing behavior with a computerized video tracking system (see Materials and Methods).

\section{Histological analysis}

Before the experiments, rats were bilaterally implanted with intracranial infusion cannulas for temporarily inactivating the
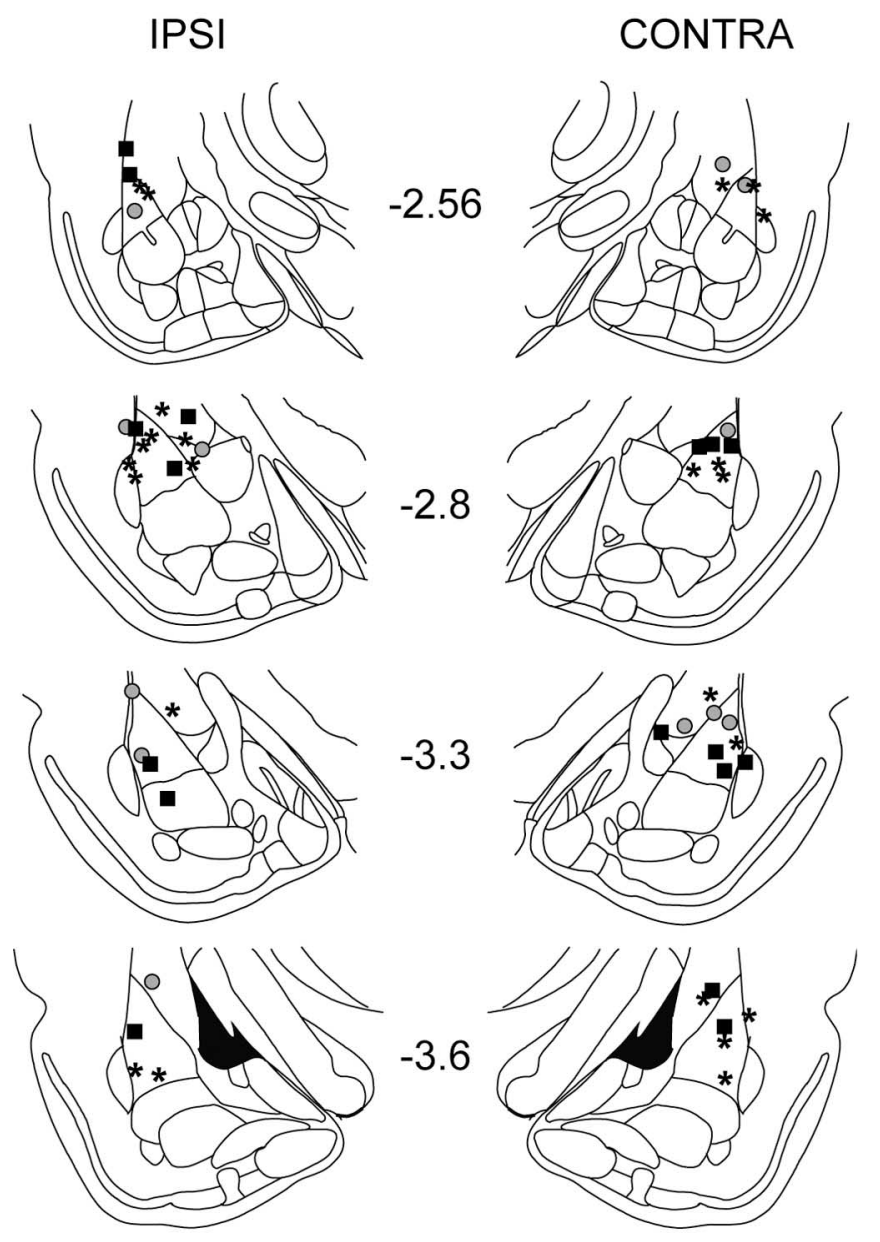

Figure 1. Histological analysis of cannula placements. Locations of cannula tip placements where muscimol was delivered are shown (control placements where no drugs were delivered are omitted for clarity). All placement hemispheres are specified relative to the eyelid where the shock US was delivered during training. Squares indicate unilateral groups (IPSI/CONTRA), circles indicate the BI group, and asterisks indicate the PAIR group.

amygdala with the $\mathrm{GABA}_{\mathrm{A}}$ agonist muscimol (see Materials and Methods). Cannula tips were targeted at the LA, and postmortem histology (Fig. 1) showed that most placements were in the targeted area, although some were located very near the LA in adjacent areas such as the amygdalostriatal transition area, basal nucleus (B), and central nucleus of the amygdala (CeA). Only placements that were $<0.25 \mathrm{~mm}$ away from the LA were included in the study (four animals were dropped from the study and replaced because of incorrect cannula placements). The volume of each infusion was $0.5 \mu \mathrm{l} /$ hemisphere, and the concentration was $0.25 \mathrm{mg} / \mathrm{ml}$ drug dissolved in $0.9 \%$ saline vehicle. This infusion volume and drug concentration may have inactivated portions not only of the LA but also of the basal and central nuclei, because muscimol is known to diffuse into areas beyond the injector tip (Edeline et al., 2002). To control for spread of muscimol into other brain areas, an off-site control group was implanted in the caudate nucleus overlying the amygdala (see Fig. 4). The behavioral results reported here were not related in any detectable way to variations in cannula placements.

\section{Experiment 1: acquisition of fear conditioning}

To investigate lateralization of the role of the amygdala in fear acquisition, rats were given pretraining infusions of muscimol to inactivate the amygdala before CS-US pairings. The rats were 
divided into five groups: a bilateral (BI; $n=6)$ group in which both amygdala hemispheres were inactivated; an ipsilateral (IPSI; $n=8$ ) group in which the amygdala ipsilateral to the shocked eyelid was inactivated; a contralateral (CONTRA; $n=8$ ) group in which the amygdala contralateral to the shocked eyelid was inactivated; a paired drug-free control group (PAIR; $n=12$ ), which received no infusions; and an unpaired drug-free control group (UNPAIR; $n=6$ ), which received no infusions and was trained with explicitly unpaired presentations of the CS and US (rather than paired CS-US presentations, as in all other groups). The shock US was always delivered to the same eyelid throughout training, with one-half of the rats in each group receiving shocks to the left eyelid and the other half receiving shocks to the right eyelid.

Figure $2 a$ illustrates the design of the experiment. On day 1, rats received six baseline test trials in which the CS was presented alone. Freezing behavior was measured during the $20 \mathrm{~s} \mathrm{CS}$ and pre-CS periods to establish baseline freezing levels (Fig. 2b). A two-factor ANOVA showed that during the preconditioning baseline session, freezing levels did not significantly depend on experimental group $\left(F_{(4,35)}=0.33 ; p=0.85\right)$, stimulus (pre-CS vs CS; $\left.F_{(1,35)}=1.21 ; p=0.28\right)$, or interaction between group and stimulus $\left(F_{(4,35)}=1.19 ; p=0.33\right)$. After the baseline session, rats were removed from the experimental platform and given muscimol infusions according to their group assignments (BI, IPSI, or CONTRA; no infusions in the PAIR and UNPAIR groups). Twenty minutes later, rats were returned to the platform for 16 training trials in which both the CS and US were presented. On day 2, rats were given six drug-free expression test trials, and freezing responses were measured during the pre-CS and CS periods (Fig. 2c).

An ANOVA with two factors (session and group) showed that the degree of conditioned freezing to the CS (Fig. 2b,c, filled bars) varied significantly among groups (group by session interaction; $\left.F_{(4,35)}=4.47 ; p=0.005\right)$. Pairwise comparisons using Fisher's least significant difference (LSD) test (Table 1) showed that only the rats in the PAIR and IPSI groups acquired significant conditioned freezing to the CS, whereas conditioned freezing was absent in the UNPAIR, BI, and CONTRA groups.

Rats were tested in the same chamber where they were trained, so fear of the training context may have contributed to the freezing that was observed during the CS period. To obtain a more selective measure of CS-evoked freezing, we subtracted each rat's freezing score during the pre-CS period from the freezing score during the CS period in each session, to remove the contextual component of the freezing score. Figure $2 d$ shows that CSspecific freezing was negligible during baseline sessions for all groups. During the test session, CS-specific freezing was apparent only in the PAIR and IPSI groups. Confirming this, a two-way ANOVA of CS minus pre-CS freezing revealed a significant main effect of session (baseline vs test; $F_{(1,35)}=8.9415 ; p=0.005076$ ), a significant main effect of group $\left(F_{(4,35)}=5.3484 ; p=0.00181\right)$, and a significant group by session interaction $\left(F_{(4,35)}=5.9833\right.$; $p=0.0009)$. Post hoc comparisons with Fisher's LSD test showed that after conditioning, CS-specific freezing was significantly elevated from baseline in the PAIR $(p=0.00002)$ and IPSI $(p=$ $0.00036)$ groups but not in the UNPAIR $(p=0.2484), \mathrm{BI}(p=$ $0.7456)$, and CONTRA ( $p=0.4817$ ) groups.

In summary, all of our statistical analyses support the conclusion that rats in the PAIR and IPSI groups acquired conditioned freezing responses to the CS, whereas rats in other groups did not. It is not surprising that rats in the UNPAIR group failed to acquire conditioned freezing to the CS, because the CS was not paired with shock in this group. Likewise, it is not surprising that rats in the BI group failed to acquire freezing to the CS, because previous studies have shown that bilateral amygdala inactivation blocks the acquisition of auditory fear conditioning (Helmstetter and Bellgowan, 1994; Muller et al., 1997; Wilensky et al., 1999). However, it is surprising that rats in the CONTRA group failed to acquire freezing to the CS, because previous studies have shown that unilateral disruption of the amygdala does not block acquisition of fear conditioning when a foot shock is used as the US (LaBar and LeDoux, 1996; Goosens and Maren, 2001; Baker and Kim, 2004). Consistent with these previous findings, rats in the IPSI group of experiment 1 were not impaired in auditory fear conditioning. Why then did rats in the CONTRA group fail to acquire freezing to the CS? Rats in the CONTRA group were retrained drug-free after the expression test on day 2, and they exhibited normal conditioned freezing to the CS when retested on day 3 (two-by-two repeated-measures ANOVA: baseline vs day 3 test session, $F_{(1,7)}=9.56, p=0.017$; pre-CS vs CS, $F_{(1,7)}=$ 23.23, $p=0.002$; session by stimulus interaction, $F_{(1,7)}=23.32$, $p=0.002)$. Thus, CONTRA rats did not sustain permanent impairment of fear conditioning after muscimol infusions on day 1 . The findings of experiment 1 therefore demonstrate that acquisition of conditioned freezing to a CS paired with unilateral eyelid shock depends only on the amygdala contralateral from the shocked eyelid and not on the ipsilateral amygdala.

\section{Comparing the aversiveness of left versus right eyelid shocks}

To examine whether left and right eyelid shocks were similarly aversive to the rats, we pooled data from rats in the PAIR $(n=12)$ and IPSI ( $n=8$ ) groups ( $n=20$ in both groups combined). Both of these groups acquired significant freezing responses to the CS, as explained above. One-half of the pooled rats $(n=10)$ were shocked on the left eyelid during training, and the other half $(n=$ 10) were shocked on the right eyelid. A three-factor ANOVA with eyelid, session (baseline vs test), and stimulus (pre-CS vs CS) as factors revealed no significant main effect of eyelid $\left(F_{(1,18)}=\right.$ $0.556 ; p=0.47)$, nor was there a significant interaction between eyelid and session $\left(F_{(1,18)}=0.523 ; p=0.48\right)$ or eyelid and stimulus $\left(F_{(1,18)}=0.267 ; p=0.61\right)$. Thus, left and right eyelid shocks were similarly effective at serving as the US for fear conditioning.

\section{Experiment 2: expression of fear conditioning}

To investigate lateralization of the role of the amygdala in fear expression, we used the rats from the PAIR group in experiment 1 above $(n=12)$, which had been trained drug-free and therefore had acquired normal conditioned freezing responses to the CS (Fig. $2 c, d$ ). Before running expression tests, we overtrained these rats by giving them an additional $6 \mathrm{~d}$ of training after experiment 1 was completed (Fig. $3 a$ ). Each day of overtraining consisted of a test session ( six CS-alone trials) followed by a training session (10 CS-US pairings). During overtraining sessions, one-half of the rats $(n=6)$ always received the shock US on the left eyelid, and the other half $(n=6)$ always received the shock US on the right eyelid, as in experiment 1 . We analyzed freezing responses separately for these two groups of rats (left vs right eyelid shocks). Figure $3 a$ shows that rats in both groups exhibited robust CSevoked freezing responses during test sessions on days $2-8$, and freezing levels were not significantly different between the left and right eyelid groups on these days $\left(F_{(1,10)}=0.064 ; p=0.80\right)$.

Because CS-evoked freezing remained stable during test sessions across multiple days of training in both groups of rats, we concluded that it would be appropriate to perform repeated freezing tests on different days, with different amygdala hemi- 

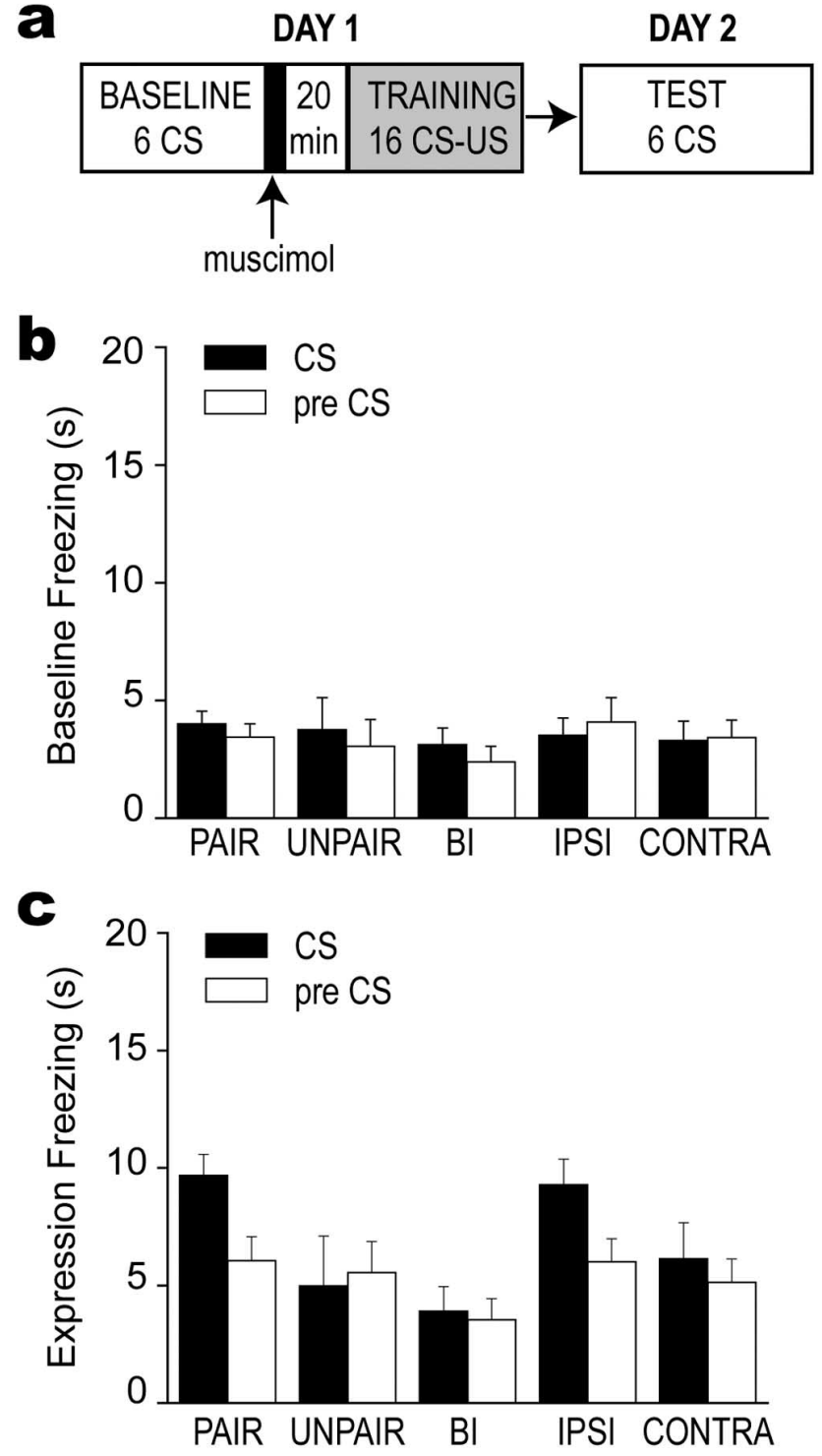

d

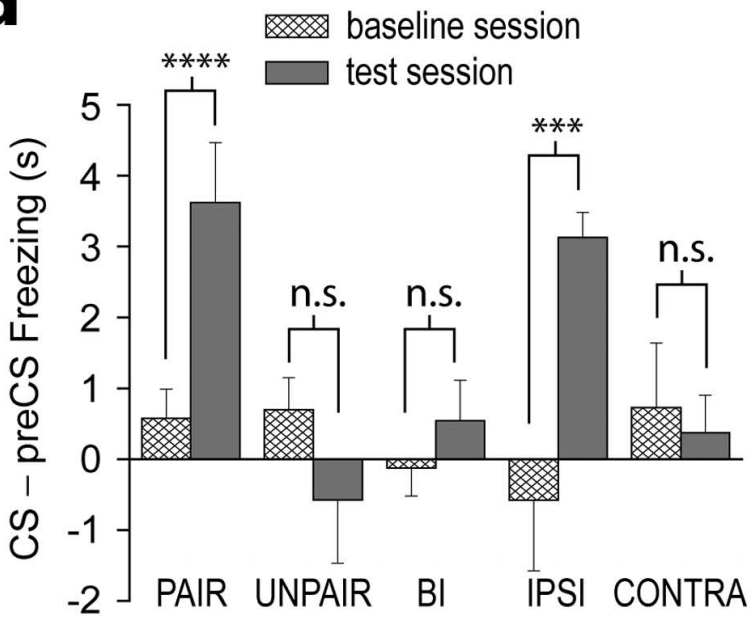

Figure 2. Acquisition. $\boldsymbol{a}$, Design of the acquisition experiment. $\boldsymbol{b}$, Mean freezing scores (seconds of freezing) during the $20 \mathrm{~s} C S$ and pre-CS periods for each group of rats during the six trials of the baseline session. $c$, Mean freezing scores during the six trials of the expression test session. $\boldsymbol{d}$, Mean freezing attributable specifically to the $C S$ and not the training context (CS minus pre-CS freezing; see Results). Significance levels for Fisher's LSD tests: ${ }^{* * *} p<0.0001$; ${ }^{* * *} p<0.001 ;$ n.s., not significant. spheres inactivated on each day. Therefore, on day 9 of the experiment, the left amygdala hemisphere was inactivated with muscimol, and rats were then given a standard test session (six CSalone trials). Figure $3 a$, leftmost shaded region, shows that left amygdala inactivation had little effect on CS-evoked freezing in the left eyelid group, but freezing was impaired in the right eyelid group. On day 10, the rats were given 10 training trials to compensate for any extinction that might have occurred during the test session on day 9. On day 11, the right amygdala hemisphere was inactivated, and rats were given another standard test session. Figure $3 a$ shows that the effect of inactivating the right amygdala on day 11 caused impairment of freezing in the left eyelid group but not the right eyelid group, exactly the opposite from what had occurred when the left amygdala was inactivated on day 9. On day 12 , the rats again received 10 training trials (as on day 10) to compensate for any extinction that might have occurred during the test session on the previous day. On day 13, the amygdala was bilaterally inactivated before testing, and impaired freezing was observed in both the left and right eyelid groups. Thus, expression of freezing was impaired when the amygdala was inactivated bilaterally or contralateral to the trained eyelid but not ipsilateral to the trained eyelid (see below for statistical analysis).

On day 14, rats were given 16 trials of reversed training during which the CS was paired with a shock US delivered to the opposite eyelid from the previously trained eyelid. That is, rats in the left eyelid group were trained for the first time on the right eyelid, and rats in the right eyelid group were trained for the first time on the left eyelid. On day 15 , the amygdala was unilaterally inactivated in the hemisphere contralateral from the originally trained eyelid in each group (i.e., the right amygdala was inactivated in the left eyelid group, and the left amygdala was inactivated in the right eyelid group). Figure $3 a$ shows that, unlike previously (day 11 for the left eyelid group and day 9 for the right eyelid group), inactivation of the amygdala contralateral to the originally trained eyelid no longer impaired freezing in either group on day 15 . Hence, a single day of reversed training on the opposite eyelid was sufficient to rescue the expression deficit caused by inactivation of the amygdala contralateral from the originally trained eyelid.

To quantify the effects of amygdala inactivation on expression of freezing to the CS, we ran a two-way ANOVA with group (left eyelid vs right eyelid) and inactivation hemisphere (left, right, bilateral, and contralateral after reverse training) as factors. The dependent variable was CS-evoked freezing during each test session, plotted in Figure $3 a$, shaded regions. This analysis revealed a highly significant interaction between group and hemisphere $\left(F_{(3,30)}=6.14 ; p=0.0022\right)$, showing that inactivation of the left and right amygdala hemispheres had very different effects on freezing depending on whether rats had been trained on the left or right eyelid. To further confirm this result, we pooled the data from the left and right eyelid groups and analyzed freezing expression during ipsilateral, contralateral (before and after reversed eyelid training), and bilateral amygdala inactivation for both groups combined. Figure $3 d$ shows that CS-evoked freezing is evident only during ipsilateral amygdala inactivation and during contralateral inactivation after reversed eyelid training (main effect of inactivation condition, $F_{(3,33)}=5.42, p=0.004$; main effect of stimulus, $F_{(1,11)}=16.33, p=0.002$; inactivation by stimulus interaction, $\left.F_{(3,33)}=12.99, p=0.000009\right)$. Multiple comparisons using Fisher's LSD test verified that expression of CS-evoked freezing was impaired when the amygdala was inactivated contralateral but not ipsilateral to the training eyelid, and reversed eyelid training rescued the deficit caused by contralateral amygdala inactivation. Table 2 also shows that CS-evoked 
Table 1. Fisher's LSD comparisons of freezing during the CS period in the acquisition experiment

\begin{tabular}{|c|c|c|c|c|c|c|c|c|c|c|}
\hline & \multicolumn{2}{|l|}{ PAIR } & \multicolumn{2}{|l|}{ UNPAIR } & \multicolumn{2}{|l|}{$\mathrm{BI}$} & \multicolumn{2}{|l|}{ IPSI } & \multicolumn{2}{|l|}{ CONTRA } \\
\hline & Base & Test & Base & Test & Base & Test & Base & Test & Base & Test \\
\hline \multicolumn{11}{|l|}{ PAIR } \\
\hline Base & & $* * * * *$ & NS & NS & NS & NS & NS & $* * *$ & NS & NS \\
\hline Test & 0.000002 & & $* * *$ & $* *$ & $* * * *$ & $* *$ & $* * * *$ & NS & $* * *$ & $* *$ \\
\hline \multicolumn{11}{|l|}{ UNPAIR } \\
\hline Base & 0.793027 & 0.000123 & & NS & NS & NS & NS & $* *$ & NS & NS \\
\hline Test & 0.615440 & 0.007803 & 0.387005 & & NS & NS & NS & * & NS & NS \\
\hline \multicolumn{11}{|l|}{$\mathrm{Bl}$} \\
\hline Base & 0.535139 & 0.000028 & 0.755779 & 0.289391 & & NS & NS & $* * *$ & NS & NS \\
\hline Test & 0.838227 & 0.001553 & 0.929410 & 0.600689 & 0.575749 & & NS & $* *$ & NS & NS \\
\hline \multicolumn{11}{|l|}{ IPS| } \\
\hline Base & 0.663573 & 0.000017 & 0.900460 & 0.372147 & 0.835559 & 0.809786 & & $* * * *$ & NS & NS \\
\hline Test & 0.000479 & 0.691737 & 0.001179 & 0.029115 & 0.000342 & 0.007466 & 0.000032 & & $* * *$ & $*$ \\
\hline \multicolumn{11}{|l|}{ CONTRA } \\
\hline Base & 0.573258 & 0.000010 & 0.814983 & 0.308527 & 0.921429 & 0.713008 & 0.906287 & 0.000189 & & NS \\
\hline Test & 0.708519 & 0.002590 & 0.549159 & 0.898190 & 0.324871 & 0.665661 & 0.421625 & 0.013664 & 0.246433 & \\
\hline
\end{tabular}

freezing during ipsilateral amygdala inactivation was slightly larger than during contralateral inactivation after reversed eyelid training ( $p=0.035355)$, which can be attributed to the fact that rats received many days of training on the original eyelid before the ipsilateral activation test but only $1 \mathrm{~d}$ of training on the reversed eyelid before the second contralateral inactivation test.

In summary, results of experiment 2 show that when the auditory CS was paired with a shock US to one eyelid, expression of conditioned freezing depended only on the amygdala contralateral from the previously shocked eyelid and not on the ipsilateral amygdala.

\section{Off-site controls}

Because muscimol can spread well beyond the injection site (Edeline et al., 2002), we ran a series of off-site controls to determine whether the effects of intra-amygdala muscimol infusions might be attributable to inactivation of brain structures other than the amygdala. Six rats were implanted bilaterally with periorbital shock electrodes and unilaterally (three left and three right) with infusion cannulas whose injector tips were targeted at the caudate nucleus $2 \mathrm{~mm}$ above the dorsal tip of the lateral amygdala. One rat had to be dropped from the study because of a problem with the periorbital shock electrode implant, which prevented it from receiving shocks on the eyelid contralateral from the cannulated hemisphere. Cannula placements for the five rats that were included in the study are shown in Figure $4 a$.

The design of the off-site control experiment is illustrated in Figure $4 b$. On day 1 , rats were given six baseline (CS-alone) trials, followed by infusion of muscimol into the caudate control site (same dosage and volume that was used for amygdala infusions above). Fifteen minutes after the infusion, the rats were given 16 CS-US pairings, with the US delivered to the eyelid contralateral from the infused hemisphere. Figure $4 c$ shows that rats froze to the CS during six drug-free test trials conducted $24 \mathrm{~h}$ after training (two-by-two repeated-measures ANOVA: baseline vs first test session, $F_{(1,4)}=16.48, p=0.0154$; pre-CS vs CS, $F_{(1,4)}=2.68$, $p=0.177$; session by stimulus interaction, $F_{(1,4)}=8.77, p=$
0.0415). This shows that infusions into the control site did not block acquisition of conditioned freezing to the CS. Immediately after these six test trials, the rats received an additional eight CS-US pairings, with the shock delivered to the same eyelid as before (to protect against any extinction that may have occurred during the six test trials). On the following day, muscimol was again infused into the caudate control site, and 15 min later, the rats were given an expression test consisting of six CS-alone presentations. Figure $4 c$ shows that rats exhibited normal expression of CS-evoked freezing after muscimol infusions into the caudate control site (two-by-two repeated-measures ANOVA: baseline vs second test session, $F_{(1,4)}=5.79, p=0.074$; pre-CS vs CS, $F_{(1,4)}=$ $12.12, p=0.0253$; session by stimulus interaction, $F_{(1,4)}=14.04$, $p=0.02)$.

In summary, muscimol infusions into the caudate control site contralateral from the shocked eyelid did not interfere with acquisition or expression of conditioned freezing to the CS. This supports the conclusion that the impairments observed in experiments 1 and 2 after infusions into the contralateral amygdala were attributable to inactivation of the amygdala region itself and not other surrounding brain structures. This is to be expected, because numerous studies have previously demonstrated the critical role of the amygdala in both acquisition and expression of fear conditioning (Maren, 2001). 
Table 2. Fisher's LSD comparisons of freezing in the expression experiment

\begin{tabular}{|c|c|c|c|c|c|c|c|c|}
\hline & \multicolumn{2}{|l|}{ IPSI } & \multicolumn{2}{|l|}{ CONTRA } & \multicolumn{2}{|l|}{ R-CONT } & \multicolumn{2}{|l|}{$\mathrm{BI}$} \\
\hline & Pre-CS & $C S$ & Pre-CS & $C S$ & Pre-CS & $C S$ & Pre-CS & CS \\
\hline \multicolumn{9}{|l|}{ IPSI } \\
\hline Pre-CS & & ** & NS & NS & NS & $* *$ & NS & NS \\
\hline CS & 0.000000 & & $* *$ & $* *$ & $* *$ & * & $* *$ & $* *$ \\
\hline \multicolumn{9}{|l|}{ CONTRA } \\
\hline Pre-CS & 0.276192 & 0.000000 & & NS & NS & $* *$ & NS & NS \\
\hline CS & 0.913793 & 0.000000 & 0.232485 & & NS & $* *$ & NS & NS \\
\hline \multicolumn{9}{|l|}{ R-CONT } \\
\hline Pre-CS & 0.539988 & 0.000000 & 0.628794 & 0.471529 & & $* *$ & NS & NS \\
\hline CS & 0.000004 & 0.035355 & 0.000000 & 0.000005 & 0.000001 & & $* *$ & $* *$ \\
\hline \multicolumn{9}{|l|}{$\mathrm{Bl}$} \\
\hline Pre-cS & 0.799520 & 0.000000 & 0.400781 & 0.717356 & 0.718737 & 0.000002 & & NS \\
\hline CS & 0.850282 & 0.000000 & 0.203459 & 0.935807 & 0.424015 & 0.000007 & 0.658316 & \\
\hline
\end{tabular}

Raw $p$ values for each comparison are listed below the diagonal. $R$, Reversed. Symbols above the diagonal denote significance levels as follows: NS, Not significant; ${ }^{* *} p<0.05 ;{ }^{* *} p<0.00001$.

\section{Discussion}

Many previous studies have shown that the amygdala is critical for pavlovian fear conditioning (for review, see by Maren, 2001). In the present study, rats were trained to fear an auditory CS by pairing it with an electric shock US delivered to one eyelid. We found that acquisition and expression of conditioned freezing to the CS depended exclusively on the amygdala contralateral from the shocked eyelid and not on the ipsilateral amygdala. This shows for the first time that the fear-conditioning circuitry of the amygdala is functionally lateralized according to the anatomical source of predicted threats. In contrast with our present results, previous rodent studies have reported that both amygdala hemispheres contribute equally to auditory fear conditioning (LaBar and LeDoux, 1996; Goosens and Maren, 2001; Baker and Kim, 2004), but all of these previous studies used a bilateral foot shock as the US, so the effect of varying the anatomical location of the US was not considered.

Our results suggest that the fear-conditioning circuitry of the amygdala is functionally lateralized according to which side of the body (left or right) a predicted threat is anticipated on. Hemispheric lateralization of learning functions has also been observed in the cerebellar eyeblink circuit, another aversive pavlovian conditioning system (Christian and Thompson, 2003). Specifically, acquisition and expression of the pavlovian eyeblink conditioned response (CR) depends on the cerebellum ipsilateral but not contralateral from the trained eyelid (McCormick et al., 1982). However, eyeblink conditioning differs from fear conditioning in several important ways (Medina et al., 2002). One major difference is that the eyeblink CR is a conditioned motor reflex controlled by the cerebellum, and the CR is anatomically confined to the same eyelid where shock is delivered during training. In contrast, the freezing CER is an emotional response controlled by the amygdala, which is thought to reflect an internal state of fear, and the freezing response is not anatomically lateralized because it is characterized by immobility of the entire musculature (Blanchard and Blanchard, 1969), regardless of where the shock is delivered during training. Here we have shown that a nonlateralized emotional response (freezing) can be controlled by different populations of neurons in opposite hemispheres of the amygdala when a threat is anticipated on the left versus right side of the body. We have shown this to be true for unilateral shocks to the eyelid, but further study is necessary to determine whether amygdala function becomes similarly lateralized when animals learn to anticipate unilateral threats to other body regions.

The role of the amygdala in aversive learning has been extensively investigated, but the sensory pathways that convey aversive signals to the amygdala remain poorly understood (Shi and Davis, 1999; Brunzell and Kim, 2001; Lanuza et al., 2004). In the present study, fear acquisition depended only on the amygdala contralateral from the shocked eyelid, implying that US pathways that convey the shock from the eyelid to the amygdala are lateralized to target the amygdala contralateral but not ipsilateral from the shock. Nociceptive signals from the eyelid enter the brain through the ipsilateral trigeminal nucleus of the medullary dorsal horn, which sends weak projections directly to the B and CeA nuclei of the contralateral amygdala (Cliffer et al., 1991; Guariau and Bernard, 2004). In addition to this weak direct pathway, the trigeminal nucleus sends strong (predominantly contralateral) projections to the posterior intralaminar thalamus (PIT) (Cliffer et al., 1991; Guariau and Bernard, 2004), which in turn sends uncrossed projections to several amygdala subnuclei (LeDoux et al., 1987, 1990a,b). Stimulation and lesion studies have suggested that the PIT might relay US signals to the amygdala (Cruikshank et al., 1992; Shi and Davis, 1999), but recent evidence indicates that these US signals may be carried by fibers of passage through the PIT rather than neurons residing within the PIT itself (Lanuza et al., 2004). Other possible US pathways from the trigeminal nucleus that might selectively target the contralateral amygdala include projections passing through the parabrachial complex to the CeA (Bernard et al., 1995; Feil and Herbert, 1995; Saper, 1995; Jasmin et al., 1997) and through the pars compacta of the ventral posterior medial thalamus to several amygdala subnuclei (Ottersen and Ben-Ari, 1979; Turner and Herkenham, 1991; Iwata et al., 1992; Guariau and Bernard, 2004). The amygdala also may receive nociceptive inputs from the insular, anterior cingulate, and perirhinal cortices (Cassell and Wright, 1986; McDonald, 1998; Shi and Cassell, 1999), and some of these cortical areas may contribute to pain processing that controls aversive learning (Shi and Davis, 1999; Johansen and Fields, 2004). However, the left and right cortical hemispheres are interconnected by many commissural pathways, so it is difficult to assess whether cortical inputs to the amygdala might convey shock signals preferentially from the contralateral but not ipsilateral eyelid.

It has been hypothesized that the amygdala (especially the LA) may be a site where sensory information about the CS and US converges to trigger synaptic plasticity that stores memories of the CS-US association during fear conditioning (for review, see Blair et al., 2001). If shock signals from one eyelid are conveyed to the contralateral but not ipsilateral LA by lateralized US pathways, then CS-US convergence might occur exclusively in the LA contralateral from the US. If so, then storage and retrieval of the CS-US association would require only the contralateral LA, and 
a

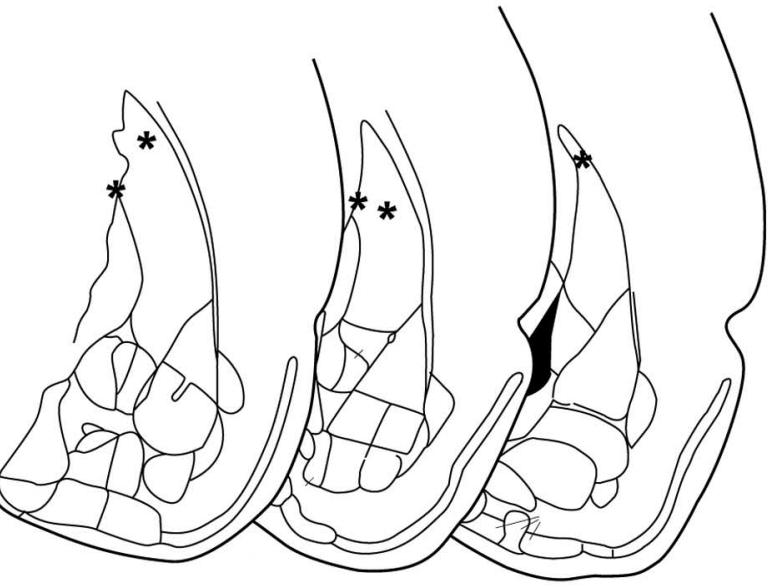

b
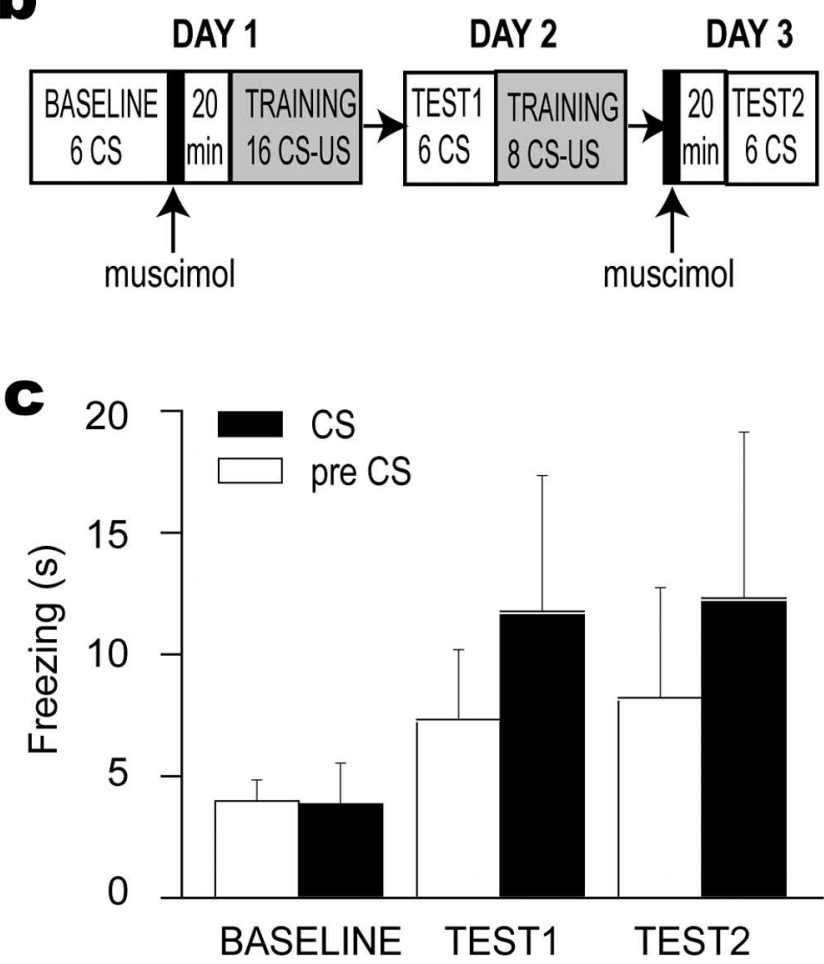

Figure 4. Off-site control injections. $\boldsymbol{a}$, Asterisks mark the site of muscimol injections into the caudate nucleus in the hemisphere contralateral from the shocked eyelid. $\boldsymbol{b}$, Design of the experiment. c, Mean freezing scores for baseline and two test sessions. TEST1 was conducted drug-free $1 \mathrm{~d}$ after training with muscimol, and TEST2 was conducted with muscimol $1 \mathrm{~d}$ after drug-free training.

both acquisition and expression of fear conditioning would depend exclusively on the contralateral amygdala as observed in our study. However, there are direct commissural connections between the left and right hemispheres of the amygdala, including the LA (Pitkanen et al., 1995; Savander et al., 1995). If US signals are conveyed through these commissural pathways, then CS-US convergence should occur in both amygdala hemispheres even when a bilateral CS is paired with a unilateral US. If this were the case, then fear acquisition might depend only on the amygdala contralateral from the US (because the US enters the amygdala via lateralized pathways targeting the contralateral side), but fear expression should be equally dependent on both amygdala hemispheres (because CS-US convergence would occur on both sides during drug-free training, and, subsequently, either amygdala hemisphere should be sufficient to retrieve the association). In conflict with this prediction, we found that both acquisition and expression of fear conditioning depended exclusively on the contralateral amygdala. This discrepancy implies that US signals from the eyelid may not pass through the commissural pathways of the amygdala to drive learning in the contralateral amygdala.

Some theorists have argued that the amygdala is not a site where memories of the CS-US association are stored during fear conditioning but, instead, that the amygdala modulates the consolidation of such associations in other brain structures (Cahill et al., 1999). If this consolidation theory is correct, then our findings imply that when rats learn to fear an auditory CS that predicts a unilateral eyelid shock, storage of the CS-US association is consolidated by the amygdala contralateral but not ipsilateral from the shocked eyelid (because acquisition of freezing was impaired only by contralateral amygdala inactivation). To account for why amygdala lesions can impair expression as well as acquisition of aversively conditioned freezing responses, the consolidation theory proposes that amygdala lesions may impair performance of the freezing response without impairing memory of the CS-US association (Vazdarjanova et al., 2001). If so, then a possible explanation for the lateralized expression deficits we observed could be that the amygdala exerts unilateral control over the performance of conditioned freezing behavior when rats anticipate a unilateral shock to one eyelid.

In conclusion, although there is controversy over exactly what role the amygdala plays in fear conditioning (Cahill et al., 1999; Fanselow and LeDoux, 1999), our results demonstrate for the first time that this role is performed by the contralateral but not ipsilateral hemisphere of the amygdala when animals learn to anticipate a shock to one eyelid. This discovery reveals that the fear-conditioning circuitry of the amygdala is functionally lateralized according to the anatomical source of predicted threats, a fact that might be used to great advantage in future studies because the ipsilateral amygdala can be used as an internal control for investigating memory-related processes that occur mainly in the contralateral amygdala. Further studies of lateralized fear conditioning may thus help identify the sensory pathways by which aversive signals reach the amygdala, elucidate the role of the amygdala in storing associative memories during aversive conditioning, and demonstrate how the amygdala governs the performance of conditioned and unconditioned defensive behaviors.

\section{References}

Baker KB, Kim JJ (2004) Amygdalar lateralization in fear conditioning: evidence for greater involvement of the right amygdala. Behav Neurosci 118:15-23.

Bernard JF, Dallel R, Raboisson P, Villanueva L, Le Bars D (1995) Organization of the efferent projections from the spinal cervical enlargement to the parabrachial area and periaqueductal gray: a PHA-L study in the rat. J Comp Neurol 353:480-505.

Blair HT, Schafe GE, Bauer EP, Rodrigues SM, LeDoux JE (2001) Synaptic plasticity in the lateral amygdala: a cellular hypothesis of fear conditioning. Learn Mem 8:229-242.

Blanchard DC, Blanchard RJ (1969) Crouching as an index of fear. J Comp Physiol Psychol 67:370-375.

Brunzell DH, Kim JJ (2001) Fear conditioning to tone, but not to context, is attenuated by lesions of the insular cortex and posterior extension of the intralaminar complex in rats. Behav Neurosci 115:365-375.

Cahill L, Weinberger NM, Roozendaal B, McGaugh JL (1999) Is the amygdala a locus of "conditioned fear"? Some questions and caveats. Neuron 23:227-228.

Cahill L, Haier RJ, White NS, Fallon J, Kilpatrick L, Lawrence C, Potkin SG, Alkire MT (2001) Sex-related difference in amygdala activity during emotionally influenced memory storage. Neurobiol Learn Mem 75:1-9.

Cahill L, Uncapher M, Kilpatrick L, Alkire MT, Turner J (2004) Sex-related 
hemispheric lateralization of amygdala function in emotionally influenced memory: an FMRI investigation. Learn Mem 11:261-266.

Canli T, Zhao Z, Desmond J, Zhao Z, Gabrieli J (2002) Sex differences in the neural basis of emotional memories. Proc Natl Acad Sci USA 99:10789-10794.

Cassell MD, Wright DJ (1986) Topography of projections from the medial prefrontal cortex to the amygdala in the rat. Brain Res Bull 17:321-333.

Christian KM, Thompson RF (2003) Neural substrates of eyeblink conditioning: acquisition and retention. Learn Mem 10:427-455.

Cliffer KD, Burstein R, Giesler Jr GJ (1991) Distributions of spinothalamic, spinohypothalamic, and spinotelencephalic fibers revealed by anterograde transport of PHA-L in rats. J Neurosci 11:852-868.

Cruikshank SJ, Edeline JM, Weinberger NM (1992) Stimulation at a site of auditory-somatosensory convergence in the medial geniculate nucleus is an effective unconditioned stimulus for fear conditioning. Behav Neurosci 106:471-483.

Davis M (1992) The role of the amygdala in fear and anxiety. Annu Rev Neurosci 15:353-375.

Edeline JM, Hars B, Hennevin E, Cotillon N (2002) Muscimol diffusion after intracerebral microinjections: a reevaluation based on electrophysiological and autoradiographic quantifications. Neurobiol Learn Mem 78:100-124.

Fanselow MS, LeDoux JE (1999) Why we think plasticity underlying Pavlovian fear conditioning occurs in the basolateral amygdala. Neuron 23:229-232.

Feil K, Herbert H (1995) Topographic organization of spinal and trigeminal somatosensory pathways to the rat parabrachial and Kolliker-Fuse nuclei. J Comp Neurol 353:506-528.

Funayama ES, Grillon CG, Davis M, Phelps EA (2001) A double dissociation in the affective modulation of startle in humans: effects of unilateral temporal lobectomy. J Cogn Neurosci 13:721-729.

Goosens KA, Maren S (2001) Contextual and auditory fear conditioning are mediated by the lateral, basal, and central amygdaloid nuclei in rats. Learn Mem 8:148-155.

Guariau C, Bernard JF (2004) A comparative reappraisal of projections from the superficial laminae of the dorsal horn in the rat: the forebrain. J Comp Neurol 468:24-56.

Helmstetter FJ, Bellgowan PS (1994) Effects of muscimol applied to the basolateral amygdala on acquisition and expression of contextual fear conditioning in rats. Behav Neurosci 108:1005-1009.

Iwata K, Kenshalo Jr DR, Dubner R, Nahin RL (1992) Diencephalic projections from the superficial and deep laminae of the medullary dorsal horn in the rat. J Comp Neurol 321:404-420.

Jasmin L, Burkey AR, Card JP, Basbaum AI (1997) Transneuronal labeling of a nociceptive pathway, the spino (trigemino)-parabrachioamygdaloid, in the rat. J Neurosci 17:3751-3765.

Johansen JP, Fields HL (2004) Glutamatergic activation of anterior cingulate cortex produces an aversive teaching signal. Nat Neurosci 7:398-403.

Klüver H, Bucy PC (1937) "Psychic blindness" and other symptoms following bilateral temporal lobectomy in rhesus monkeys. Am J Physiol 119:352-353.

Konorski J (1967) Integrative activity of the brain. Chicago: University of Chicago.

LaBar KS, LeDoux JE (1996) Partial disruption of fear conditioning in rats with unilateral amygdala damage: correspondence with unilateral temporal lobectomy in humans. Behav Neurosci 110:991-997.

Lanuza E, Nader K, Ledoux JE (2004) Unconditioned stimulus pathways to the amygdala: effects of posterior thalamic and cortical lesions on fear conditioning. Neuroscience 125:305-315.

LeDoux JE, Ruggiero DA, Forest R, Stornetta R, Reis DJ (1987) Topographic organization of convergent projections to the thalamus from the inferior colliculus and spinal cord in the rat. J Comp Neurol 264:123-146.

LeDoux JE, Iwata J, Cicchetti P, Reis DJ (1988) Different projections of the central amygdaloid nucleus mediate autonomic and behavioral correlates of conditioned fear. J Neurosci 8:2517-2529.

LeDoux JE, Farb C, Ruggiero DA (1990a) Topographic organization of neurons in the acoustic thalamus that project to the amygdala. J Neurosci 10:1043-1054.

LeDoux JE, Cicchetti P, Xagoraris A, Romanski LM (1990b) The lateral amygdaloid nucleus: sensory interface of the amygdala in fear conditioning. J Neurosci 10:1062-1069.

Maren S (2001) Neurobiology of Pavlovian fear conditioning. Annu Rev Neurosci 24:897-931.

McCormick DA, Guyer PE, Thompson RF (1982) Superior cerebellar peduncle lesions selectively abolish the ipsilateral classically conditioned nictitating membrane/eyelid response of the rabbit. Brain Res 244:347-350.

McDonald AJ (1998) Cortical pathways to the mammalian amygdala. Prog Neurobiol 55:257-332.

Medina JF, Christopher Repa J, Mauk MD, LeDoux JE (2002) Parallels between cerebellum- and amygdala-dependent conditioning. Nat Rev Neurosci 3:122-131.

Moita MA, Rosis S, Zhou Y, LeDoux JE, Blair HT (2003) Hippocampal place cells acquire location-specific responses to the conditioned stimulus during auditory fear conditioning. Neuron 37:485-497.

Moita MA, Rosis S, Zhou Y, LeDoux JE, Blair HT (2004) Putting fear in its place: remapping of hippocampal place cells during fear conditioning. J Neurosci 24:7015-7023.

Morris JS, Ohmann A, Dolan RJ (1998) Conscious and unconscious emotional learning in the human amygdala. Nature, 393:467-470.

Muller J, Corodimas KP, Fridel Z, LeDoux JE (1997) Functional inactivation of the lateral and basal nuclei of the amygdala by muscimol infusion prevents fear conditioning to an explicit CS and to contextual stimuli. Behavioral Neuroscience 111:683-691.

Ottersen OP, Ben-Ari Y (1979) Afferent connections to the amygdaloid complex of the rat and cat. J Comp Neurol 187:401-424.

Phelps EA, O'Connor KJ, Gatenby JC, Gore JC, Grillon C, Davis M (2001) Activation of the left amygdala to a cognitive representation of fear. Nat Neurosci 4:437-441.

Pitkanen A, Stefanacci L, Farb CR, Go GG, LeDoux JE, Amaral DG (1995) Intrinsic connections of the rat amygdaloid complex: projections originating in the lateral nucleus. J Comp Neurol 356:288-310.

Saper CB (1995) The spinoparabrachial pathway: shedding new light on an old path. J Comp Neurol 353:477-479.

Savander V, Go CG, LeDoux JE, Pitkanen A (1995) Intrinsic connections of the rat amygdaloid complex: projections originating in the basal nucleus. J Comp Neurol 361:345-368.

Shi C, Davis M (1999) Pain pathways involved in fear conditioning measured with fear-potentiated startle: lesion studies. J Neurosci 19:420 -430.

ShiCJ, Cassell MD (1999) Perirhinal cortex projections to the amygdaloid complex and hippocampal formation in the rat. J Comp Neurol 406:299-328.

Turner BH, Herkenham M (1991) Thalamoamygdaloid projections in the rat: a test of the amygdala's role in sensory processing. J Comp Neurol 313:295-325.

Vazdarjanova A, Cahill L, McGaugh JL (2001) Disrupting basolateral amygdala function impairs unconditioned freezing and avoidance in rats. Eur J Neurosci 14:709-718.

Wagner AR, Brandon SE (1989) Evolution of a structured model of Pavlovian conditioning (AESOP). In: Contemporary learning theories: pavlovian conditioning and the status of traditional learning theory (Klein SB, Mowrer RR, eds), pp 149-189. Hillsdale, NJ: Erlbaum.

Weiskrantz L (1956) Behavioral changes associated with ablation of the amygdaloid complex in monkeys. J Comp Physiol Psychol 49:381-391.

Wilensky AE, Schafe GE, LeDoux JE (1999) Functional inactivation of the amygdala before but not after auditory fear conditioning prevents memory formation. J Neurosci 19:RC48(1-5). 